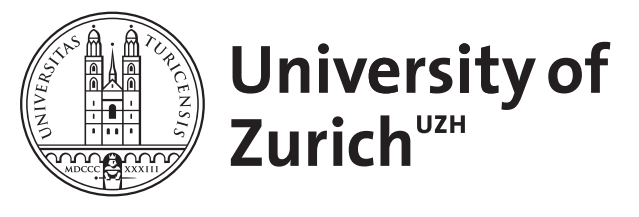

\title{
Ist die Fibromyalgie eine Viruserkrankung?
}

\author{
Sprott, H
}

\begin{abstract}
Sind Viren verantwortlich für den Schmerz bei Patienten mit Fibromyalgie? Sind Viren der Auslöser der rheumatoiden Arthritis? Ist das "chronic fatigue syndrome" eine Viruserkrankung? Viele offene Fragen - wenig oder kontroverse Antworten. Nach derzeitiger Kenntnis über die Entstehung des Schmerzes bei der Fibromyalgie lösen sowohl periphere als auch zentrale Mechanismen die "bunte" Symptomatik der Fibromyalgie aus. Trotz ihres weiten Symptomspektrums handelt es sich bei der Erkrankung jedoch um eine spezifische Entität, die hauptsächlich mit dualen Wiederaufnahmehemmern, Antikonvulsiva, Tramadol, selektiven Serotoninwiederaufnahmehemmern, Gammahydroxybutyrat und Dopaminagonisten in individuell sinnvoller Kombination $\mathrm{zu}$ behandeln ist
\end{abstract}

DOI: https://doi.org/10.1007/s00393-011-0825-3

Posted at the Zurich Open Repository and Archive, University of Zurich

ZORA URL: https://doi.org/10.5167/uzh-156502

Journal Article

Published Version

Originally published at:

Sprott, H (2011). Ist die Fibromyalgie eine Viruserkrankung? Zeitschrift für Rheumatologie, 70(8):637638.

DOI: https://doi.org/10.1007/s00393-011-0825-3 
Z Rheumatol 2011 · 70:637-638

DOI 10.1007/s00393-011-0825-3

Online publiziert: 24. Juni 2011

(c) Springer-Verlag 2011

\section{Redaktion}

U. Müller-Ladner, Bad Nauheim

U. Lange, Bad Nauheim
H. Sprott

Rheumaklinik und Institut für Physikalische Medizin, UniversitätsSpital Zürich
Von verschiedenen neuropathischen Schmerzerkrankungen kennen wir eine virale Genese. $\mathrm{Zu}$ nennen ist das Beispiel des Herpes zoster, die sensorische Neuropathie bei Hepatitis-C-Infektion oder die Beeinflussung des neuropathischen Schmerzes durch Herpes simplex Typ 1 [1, $2,3]$.

Weder die 1990er ACR-Klassifikationskriterien noch der Versuch in 2010, die Fibromyalgie simpel zu diagnostizieren, helfen bezüglich der Ätiologie der Erkrankung weiter $[4,5]$. Natürlich gibt es Berichte über die Seroprävalenz des Parvovirus B19 bei Fibromyalgie [6] bzw. des humanen T-Zell-lymphotropischen Virus Typ 1 [7], aber letztendlich gibt es mindestens genauso viele Publikationen, die dem widersprechen $[8,9]$.

Es ist durchaus verständlich, dass sowohl Health Care Professionals als auch Patienten und Patientenorganisationen daran interessiert sind, die Ursache der Fibromyalgie in Stein zu meißeln, da aufgrund der ,weichen“ Klassifikationskriterien und der immer noch fehlenden diagnostischen Kriterien die Akzeptanz der Erkrankung, selbst unter Spezialisten, eine Berg-und-Tal-Fahrt erlebt. Ein glasklarer Nachweis einer bestehenden Symptomatik, z. B. durch einen Erreger, würde hier sicherlich weiterhelfen. Diese Diskussion gibt es jedoch nicht nur beim Krankheitsbild der Fibromyalgie, sondern auch bei anderen unspezifischen und eher funktionellen Erkrankungen, wie dem ,irritable bowel syndrome“ oder auch dem "chronic fatigue syndrome" $[10]$.

Aufgrund dieser fehlenden, kausalen Argumente geht es z. B. in der Schweiz so weit, dass die Gesetzgebung die Fibromyalgie aus der Liste der „rentenwürdigen Erkrankungen" verbannt hat.
In den USA sind 3 Medikamente für die Therapie der Fibromyalgie zugelassen: die Wirkstoffe Pregabalin, Duloxetin, und Milnacipran. Pregabalin gehört zu den Antiepileptika, und Duloxetin und Milnacipran zählen zu den Antidepressiva. Es ist bekannt, dass diese Substanzgruppen effektiv bei der Behandlung von neuropathischen Schmerzen sind. Beschrieben wird bei $12 \%$ der mit Pregabalin behandelten Patienten eine Schmerzreduktion von 30\% oder mehr; bei den mit Duloxetin behandelten Patienten sind es $14 \%$ und bei den mit Milnacipran behandelten Patienten 5\%. Für die betreffenden Patienten ist dies ein großer Erfolg und mit Sicherheit ein Gewinn an Lebensqualität; für die Mehrheit jedoch bleiben diese Wirkstoffe ohne einen Benefit. Mit einem viralen Angriffspunkt hat dies nichts zu tun. In diesem Zusammenhang ist es interessant zu wissen, dass dieser "Goldstandard“ bei der Behandlung spezieller neuropathischer Schmerzen, wie z. B. bei der HIVinduzierten Polyneuropathie, nachweislich negative Evidenz besitzt.

Die derzeitige Kenntnis über die Entstehung der Schmerzen bei der Fibromyalgie besagt, dass es sowohl periphere als auch zentrale Mechanismen gibt, die, stark verallgemeinert, zu einem erhöhten Input nach zentral und zu einer gestörten Hemmung von zentral, die „bunte“ Symptomatik der Fibromyalgie auslösen. Es ist eine spezifische Entität, wenn wir auch Patienten sehen, deren Symptome ein weites Spektrum begleiten. Die Therapie ist individuell mit starker Evidenz für duale Wiederaufnahmehemmer und Antikonvulsiva, mit moderater Evidenz für Tramadol und selektive Serotoninwiederaufnahmehemmer, Gammahydroxybutyrat und Dopaminagonisten, sowie schwacher Evi- denz für Wachstumshormon, Tropisetron etc. Keinerlei Evidenz liegt vor für Opioide und Steroide sowie für Benzodiazepine [11]. Eine sinnvolle Kombinationstherapie zur Potenzierung der Wirkungen und zur Vermeidung allfälliger Nebenwirkungen sollte das Ziel jeder individuellen Therapie bei Fibromyalgiepatienten sein. Von einer antiviralen Therapie sind wir momentan meilenweit entfernt.

\section{Korrespondenzadresse \\ Prof. Dr. H. Sprott}

Rheumaklinik und Institut für Physikalische Medizin, UniversitätsSpital Zürich

Gloriastr. 25, CH-8091 Zürich

Schweiz

haiko.sprott@usz.ch

Interessenkonflikt. Der korrespondierende Autor gibt an, dass kein Interessenkonflikt besteht.

\section{Literatur}

1. Johnson RW, Bouhassira D, Kassianos G et al (2010) The impact of herpes zoster and post-herpetic neuralgia on quality-of-life. BMC Med 8:37

2. Yoon MS, Obermann M, Dockweiler C et al (2011) Sensory neuropathy in patients with cryoglobulin negative hepatitis-C infection. J Neurol 258(1):8088

3. Martins I, Costa-Araujo S, Fadel J et al (2010) Reversal of neuropathic pain by HSV-1-mediated decrease of noradrenaline in a pain facilitatory area of the brain. Pain 151:137-145

4. Wolfe F, Smythe HA, Yunus MB et al (1990) The American College of Rheumatology 1990 criteria for the classification of fibromyalgia. Report of the multicenter criteria committee. Arthritis Rheum 33:160-172

5. Wolfe F, Clauw DJ, Fitzcharles MA et al (2010) The American College of Rheumatology preliminary diagnostic criteria for fibromyalgia and measurement of symptom severity. Arthritis Care Res 62:600-610

6. Buyukkose M, Kozanoglu E, Basaran S et al (2009) Seroprevalence of parvovirus B19 in fibromyalgia syndrome. Clin Rheumatol 28:305-309 
Z Rheumatol $2011 \cdot 70: 637-638$

DOI 10.1007/s00393-011-0825-3

(c) Springer-Verlag 2011

\section{H. Sprott \\ Ist die Fibromyalgie eine Viruserkrankung?}

\section{Zusammenfassung}

Sind Viren verantwortlich für den Schmerz bei Patienten mit Fibromyalgie? Sind Viren der Auslöser der rheumatoiden Arthritis? Ist das ",chronic fatigue syndrome" eine Viruserkrankung? Viele offene Fragen - wenig oder kontroverse Antworten. Nach derzeitiger Kenntnis über die Entstehung des Schmerzes bei der Fibromyalgie lösen sowohl periphere als auch zentrale Mechanismen die „bunte" Symptomatik der Fibromyalgie aus. Trotz ihres weiten Symptomspektrums handelt es sich bei der Erkrankung jedoch um eine spezifische Entität, die hauptsächlich mit dualen Wiederaufnahmehemmern, Antikonvulsiva, Tramadol, selektiven Serotoninwiederaufnahmehemmern, Gammahydroxybutyrat und Dopaminagonisten in individuell sinnvoller Kombination zu behandeln ist.

\section{Schlüsselwörter}

Neuropathischer Schmerz - Fibromyalgie . Krankheitsursache -Symptomatik . Klassifikationskriterien

\section{Is fibromyalgia a viral disease?}

\section{Abstract}

Are viruses responsible for the pain in patients with fibromyalgia? Are viruses the trigger for rheumatoid arthritis? Is chronic fatigue syndrome a viral disease? There are many open questions with few or controversial answers. According to the current state of knowledge on the origin of the pain in fibromyalgia the varied symptomatic of fibromyalgia is triggered by peripheral as well as central mechanisms. Despite the broad spectrum of symptoms the disease is a specific entity which is mainly treated with dual reuptake inhibitors, anticonvulsives, tramadol, selective serotonin reuptake inhibitors, gammahydroxybutyrate and dopamine agonists in individually selected combinations.

\section{Keywords}

Neuropathic pain - Fibromyalgia - Disease etiology · Symptomatic · Classification criteria
7. Cruz BA, Catalan-Soares B, Proietti F (2006) Higher prevalence of fibromyalgia in patients infected with human T cell lymphotropic virus type I. J Rheumatol 33:2300-2303

8. Narvaez J, Nolla JM, Valverde J (2005) No serological evidence that fibromyalgia is linked with exposure to human parvovirus B19. Joint Bone Spine 72:592-594

9. Narvaez J, Nolla JM, Valverde-Garcia J (2005) Lack of association of fibromyalgia with hepatitis $\mathrm{C} \mathrm{vi-}$ rus infection. J Rheumatol 32:1118-1121

10. Buchwald D (1996) Fibromyalgia and chronic fatigue syndrome: similarities and differences. Rheum Dis Clin North Am 22:219-243

11. Goldenberg DL, Burckhardt C, Crofford L (2004) Management of fibromyalgia syndrome. JAMA 292:2388-2395

\section{Fachnachrichten}

\section{Ausschreibung der Deutschen Stiftung Sklerodermie}

Die Deutsche Stiftung Sklerodermie hat sich die Unterstützung aller klinischen und experimentellen Arbeiten zur Sklerodermie zum

Ziel gesetzt. Sie fördert dazu Vorhaben einzelner Personen oder auch von Arbeitsgruppen. Anträge für kurzzeitige Stipendien, Austausch von Wissenschaftlern und Forschungsvorhaben zur Verbesserung des Verständnisses von Pathogenese, Diagnostik und Therapie der Sklerodermie im allgemeinen sowie für ein spezielles Forschungsprojekt „Sklerodermie bei Kindern und Jugendlichen" und Fortbildungsmaßnahmen für Ärzte, Therapeuten und Patienten können bei der Stiftung bis zum 29. Februar 2012 gestellt werden. Diese Anträge dürfen $€ 25.000$ nicht überschreiten und sollen sich nach dem Muster der Deutschen Forschungsgemeinschaft richten. Die Seitenzahl ist auf 7 Seiten beschränkt. Ein kurzer Lebenslauf des Bewerbers sowie ein Literaturverzeichnis sollen den Antrag ergänzen. Eingereicht werden können sie unter:

Dermatologische und Poliklinik der Universität zu Köln Deutsche Stiftung Sklerodermie z. Hd. Prof. Dr. med. Thomas Krieg Joseph-Stelzmann-Str. 9 50924 Köln www.sklerodermie-stiftung.de 